\title{
A method for representing a geomagnetic total force field in a small region with special attention to discontinuities of data
}

\author{
Ken'ichi Yamazaki ${ }^{1 *}$ and Naoto Oshiman ${ }^{2}$ \\ ${ }^{1}$ Graduate School of Science, Kyoto University, Kyoto 611-0011, Japan \\ ${ }^{2}$ Disaster Prevention Research Institute, Kyoto University, Kyoto 611-0011, Japan
}

(Received January 6, 2006; Revised July 12, 2006; Accepted July 13, 2006; Online published December 25, 2006)

\begin{abstract}
In order to represent the total force field variation in a narrow region as precisely as possible, the calculation process of NOC, which is frequently used in geomagnetic field modeling, was improved. An iterative procedure was introduced to avoid the effect of data missing in some time series. The presented procedures are applied to monthly averaged data of the geomagnetic total force field during 1980-1997 in Japan. Some local changes are detected using this model. One of them may be related to an earthquake that took place near the site.
\end{abstract}

Key words: Geomagnetic field model, NOC, tectonomagnetic phenomena, missing value.

\section{Introduction}

Many kinds of geomagnetic reference field models have been made. Global models, such as the International Geomagnetic Reference Field (Mandea and Macmillan, 2000), don't have enough precision in the ocean hemisphere and its marginal regions such as the Far East (Burdelnaya et al., 1999). The reason for such a low precision arose from a biased distribution of observatories that the models rely on. Biased distribution, however, becomes of benefit to models for a restricted region but not for the whole globe. Actually, some regional models which have higher precision than global ones have been constructed (Golovkov and Newitt, 1997). The main purpose of geomagnetic models is to represent widely extended features in the geomagnetic field and eliminate local disturbances. But another application of the modeling is to extract small local changes which are not widely extended by using the model. Some parts of geomagnetic changes have their origins in the crust, and magnetization of the crust changes accompanied by the surrounding temperature or applied stress. Therefore, changes in the geomagnetic field yield information on the physical states in the crust. These changes, however, are very small in general, and are hardly recognized in the original data, since larger changes due to other effects conceal them. Geomagnetic field models are used as references for them.

The required precision for these two applications are different because of the difference between amplitudes of widely distributed changes and local ones. How to express the temporal changes is an important problem in constructing a model of high precision. Any analytical functions are inadequate to represent realistic changes, and numeri-

\footnotetext{
* Now at Earthquake Research Institute, the University of Tokyo, Tokyo 113-0032, Japan.

Copyright (c) The Society of Geomagnetism and Earth, Planetary and Space Sciences (SGEPSS); The Seismological Society of Japan; The Volcanological Society of Japan; The Geodetic Society of Japan; The Japanese Society for Planetary Sciences; TERRAPUB.
}

cal functions should be used in regional modeling (Burdelnaya et al., 1999). One method to determine such functions is the natural orthogonal component method (NOC) (Fainberg, 1975). In NOC analysis, statistical properties between the temporal changes at each site are used to estimate the basis functions for representing the geomagnetic changes. Models using this method have already made for some regions (Burdelnaya et al., 1999). The required accuracy for extracting the changes in the origin of the crust is about $1 \mathrm{nT}$. In previous models, this criterion is satisfied for some stations, but not for all. Therefore, it is necessary to construct more accurate models.

Much station data should be used to improve the accuracy of model, and some data might contain missing periods of data acquisition. However, the existence of missing values sometimes becomes an obstacle for the statistical analysis, and appropriate steps to handle these data are required. In this paper, procedures to handle the discontinuity of data are proposed and their validity is tested. The proposed procedures are applied to secular changes in the geomagnetic total force obtained in Japan, and some features of extracted local ones are reported.

\section{Method}

2.1 Natural Orthogonal Component Analysis

NOC analysis is used for describing changes commonly appearing in many sites. Here we briefly outline NOC analysis. First, the base values for each site are removed from the original data:

$$
\Delta F_{i j}=F_{i j}-b_{j}
$$

where $F_{i j}$ are the values at the $j$-th site at time $i$ and $b_{j}$ is the base value there, respectively. Then, for simplicity, let $\Delta F_{i j}$ be expressed as a matrix $\mathbf{F}$, whose $(i, j)$ th component is $\Delta F_{i j} . \mathbf{F}$ can be decomposed into two matrices, $\mathbf{T}$ and $\mathbf{X}$, as follows:

$$
\mathbf{F}=\mathbf{T X}^{T}
$$


where the number of columns of $\mathbf{T}$ and $\mathbf{X}$ are both equal to the number of observation sites, $M$. $\mathbf{T}$ and $\mathbf{X}$ can be chosen to satisfy the following orthogonal conditions:

$$
\begin{aligned}
& \mathbf{T}^{T} \mathbf{T}=\Lambda \\
& \mathbf{X}^{T} \mathbf{X}=M \mathbf{1}
\end{aligned}
$$

where $\Lambda$ is a diagonal matrix and $\mathbf{1}$ is a unit matrix. Then, multiplying the transpose of $\mathbf{F}$ from the left and $\mathbf{X}$ from the right to Eq. (2) leads to

$$
\left(\mathbf{F}^{T} \mathbf{F}\right) \mathbf{X}=M \mathbf{X} \Lambda .
$$

$\mathbf{X}$ can be obtained by solving this eigenvalue problem. For convenience, each column of $\mathbf{X}$ should be arranged as the diagonal components of $\Lambda$ is descending order. On the other hand, multiplying $\mathbf{X}$ to the right side of Eq. (2) gives T as follows:

$$
\mathbf{T}=\frac{1}{M} \mathbf{F X} .
$$

The $l$-th column of $\mathbf{T}$ is called the $l$-th NOC or temporal function. We use only left $K$ columns of matrices $\mathbf{X}$ and $\mathbf{T}$ to construct the regional model for an approximating expression for $\mathbf{F}$ as follows:

$$
\mathbf{F}=\mathbf{T}_{K} \mathbf{X}_{K}+\epsilon,
$$

where $\mathbf{X}_{K}$ and $\mathbf{T}_{K}$ are the left $K$ column of matrices $\mathbf{X}$ and $\mathbf{T}$, and $\epsilon$ is a matrix of residuals, respectively. It can be proved that $|\epsilon|^{2}$ becomes minimum for any $K$ (Fainberg, 1975). In other words, changes described by $\mathbf{T}_{K}$ and $\mathbf{X}_{K}$ with small $K$ express common changes in much data, and those described by $\epsilon$, can be regarded as minor or local changes.

Each component of $\mathbf{X}$, named the spatial function, represents the factor of each NOC at each observation site. Our objective is to divide the observed changes into common changes and local changes, and factors for common changes should vary smoothly. Therefore, the distribution of $X_{k j}$ in space should not be random but a smooth one, and this requirement is equal to the statement that $X_{k j}$ can be expressed by a linear combination of analytical functions. In this study we choose the following expression:

$$
X_{k i}=\sum_{k^{\prime}} a_{k k^{\prime}} S_{k^{\prime}}\left(\lambda_{i}, \phi_{i}\right),
$$

where $\left(\lambda_{i}, \phi_{i}\right)$ are the longitude and latitude of the $i$ th site, and $S_{k^{\prime}}$ 's are polynomials of $\lambda_{i}$ and $\phi_{i}$, such as $1, \lambda, \phi, \lambda^{2}, \ldots$, respectively. Coefficients $a_{k k^{\prime}}$ s are determined as the least-square solutions of Eq. (7) using $\mathbf{X}$ obtained from Eq. (4).

It should be noted that the mathematical orthogonality of each NOC does not necessarily reflect the physical independence of their origins. Indeed, in some cases the NOC method is used to separate time series data to some components according to their origins (Fujiwara et al., 2001), but it is only achieved with enough data. In our case, which is demonstrated in the following sections, data from small areas and limited periods are used, and the separation to the physically independent components may be incomplete. But in our case, complete separations to physically independent components are not required. Our aim of using the

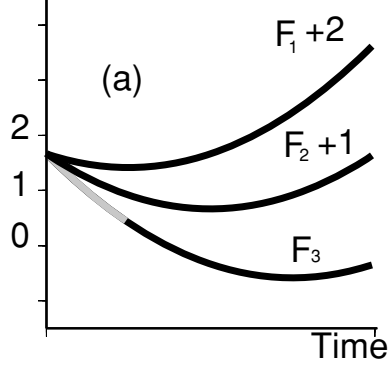

(c)

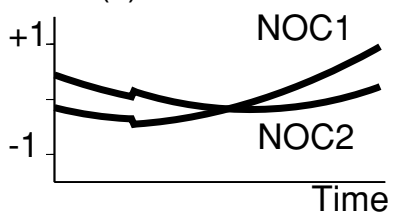

(b)

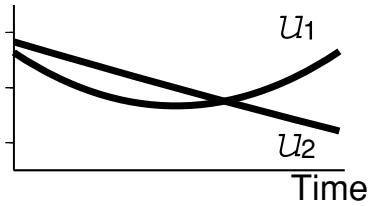

(d)

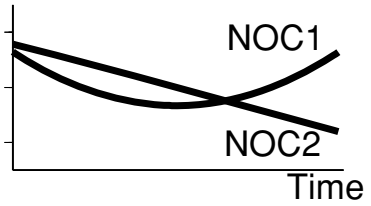

Fig. 1. An example of data and NOC analysis results for discontinuous data. Three time series shown in (a) are synthesized from the orthogonal basis functions shown in (b). $F_{1}=u_{1}-u_{2}, F_{2}=u_{1}$ and $F_{3}=u_{1}+u_{2}$, respectively. The grey line in (a) indicates missing values that are virtually included for the test. NOCs obtained from the usual procedure for handling missing values are shown in (c), and those obtained from the iterative procedure described in the text are shown in (d).

NOC procedure is not to investigate physical meanings of each NOC but to extract common changes from the data. Local changes are expected to be separated in our procedure, even if the physical independence is not completely guarranteed, because they are not only physically independent but also mathematically independent from those of regional changes.

\subsection{Handling of missing data}

When some data $F_{i j}$ are missing, the procedure described above fails because $\mathbf{F}^{T} \mathbf{F}$ cannot be obtained as such. The simplest way to resolve such an obstacle is by using only time series which contain no missing values for analysis. But since the missing data happened at most of the stations in geomagnetic observations, this way cannot be adopted for modeling in a narrow region. Another way to avoid the problem is by cutting all data acquired at the time when any one set of data is missing. However, such an exclusive cut of data causes a serious decrease in data that can be used for analysis. Therefore, some alternative ways using all the data should be adopted.

In Eq. (4), each component of matrix $\mathbf{F}^{T} \mathbf{F}$ is a covariance between each time series, and it should be determined from statistical structures between two time series. Therefore, $\mathbf{F}^{T} \mathbf{F}$ can be replaced by the matrix $\mathbf{C}$ whose components are an unbiased estimation of covariance expressed as

$$
\text { (C) })_{j j^{\prime}}=\frac{N_{e}-1}{N-1} \sum_{i} \Delta F_{i j} \Delta F_{i j^{\prime}} \text {, }
$$

where $N$ is the length of the time series, summation is taken for all time $i$ at which neither $F_{i j}$ nor $F_{i j^{\prime}}$ is missing, and $N_{e}$ is the number of such times, respectively.

In the same way as in Eq. (5), we can expect that $T_{i k}$ are 


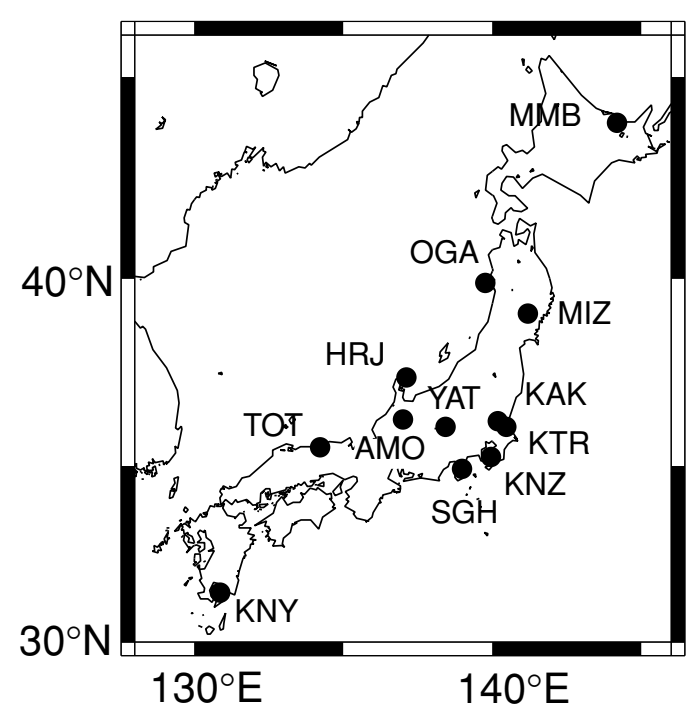

Fig. 2. Location map of the observation sites used in this study.

also obtained as the weighted sum of data $\Delta F_{i j}$ as follows:

$$
T_{i k}=\sum_{j} w_{k j}(i) \Delta F_{i j}
$$

Weighting coefficients $w$ are selected in such a way that the other NOCs will not affect the estimation of the interested NOC, and is expressed as

$$
\sum_{j} w_{k j}(i) X_{k^{\prime} j}=\delta_{k k^{\prime}} .
$$

In studies of geomagnetic field changes, daily variations are analyzed by a similar method (Kadokura, 1990).

In our case of long-term geomagnetic changes, however, the above procedure is no longer valid. Since secular changes in the geomagnetic field are smooth parts of them don't have enough information on the statistical character of the whole time series. Therefore, NOCs derived by the above steps are not expected to be correct. This expectation is confirmed by the following simple example: Fig. 1(a) shows three synthetic time series which are superpositions of the orthogonal basis functions given in Fig. 1(b). Of course, each basis function is also obtained as the result of NOC analysis for synthesized functions. When a part of them, indicated by the grey line in Fig. 1(a), is assume to be lost, the obtained NOCs (Fig. 1(c)) no longer correspond to the exact NOCs. One serious problem in these results is the step changes corresponding to the period of missing data. Models composed from these NOCs may also have these types of unreal changes, and that will lead to a misjudgement of local anomalous changes. Therefore, some advanced procedure should be applied to avoid these errors.

\subsection{Iterative process}

We suggest here an iterative process to overcome the fault of NOC analysis mentioned above. First, temporary values of NOCs, $T_{k i}^{(0)}$, and spatial functions, $X_{k j}^{(0)}$, are calculated by using the ordinary procedure of Eqs. (4), (5), and (8). Then, original data $F_{i j}$ are interpolated with these temporary val-
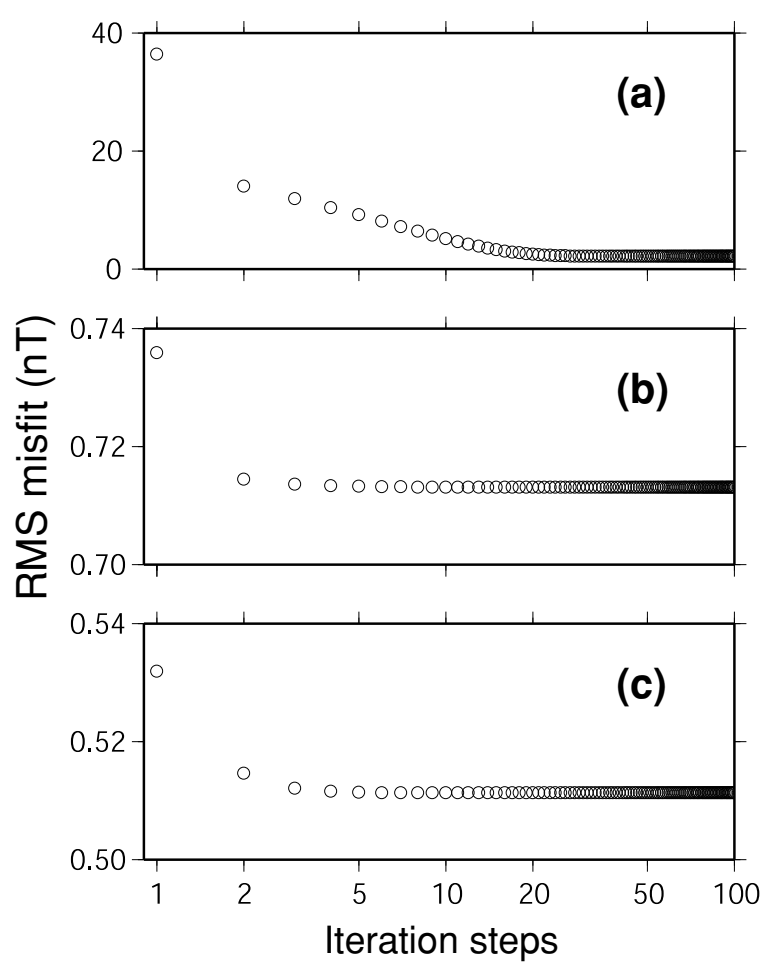

Fig. 3. RMS misfit at each iteration step in the calculation of 1st (a), 2nd (b), and 3rd (c) NOC.

ues as follows:

$$
\Delta F_{i j}^{(N+1)}= \begin{cases}\Delta F_{i j}^{(N)}+b_{j}^{(N)} & \left(\text { if } F_{i j} \text { exists }\right) \\ \sum_{k=1}^{K} T_{k i}^{(N)} X_{k j}^{(N)} & \text { (other) }\end{cases}
$$

where

$$
b_{j}^{(N)}=\sum_{i=1}^{I}\left(\sum_{k=1}^{K} T_{k i}^{(N)} X_{k j}^{(N)}\right) / N_{e}
$$

where summation is done for existing values. Then, covariant matrix $\mathbf{C}$ is recalculated using these interpolated values. Each element of $\mathbf{C}$ is expected to be closer to the real values of $\mathbf{C}$, since the base value $b_{j}$ is modified through the interpolation procedure.

This procedure can be continued by replacing the set of $\left(\mathbf{X}^{(0)}, \mathbf{T}^{(0)}\right)$ and $\left(\mathbf{X}^{(1)}, \mathbf{T}^{(1)}\right)$ by $\left(\mathbf{X}^{(N)}, \mathbf{T}^{(N)}\right)$ and $\left(\mathbf{X}^{(N+1)}, \mathbf{T}^{(N+1)}\right)$, respectively. $\left(\mathbf{X}^{(N)}, \mathbf{T}^{(N)}\right)$ are expected to converged to some limit values when $N$ goes to infinity. And it is obvious that one of its attractive points in this procedure is the set of exact values of $(\mathbf{X}, \mathbf{T})$ that we want to obtain, because decomposition of Eq. (2) for data interpolated by such a set of $(\mathbf{X}, \mathbf{T})$ should give the same values. Such values are one of the fixed points of the procedure. It may happen that $\left(\mathbf{X}^{(N)}, \mathbf{T}^{(N)}\right)$ converge to some abnormal values, but can be recognized by visual inspection of the result. In such a case, we may avoid the unexpected convergence by removing some time series from the analysis. We will demonstrate how each estimated value converges to the final result in the following section. 
(nT)

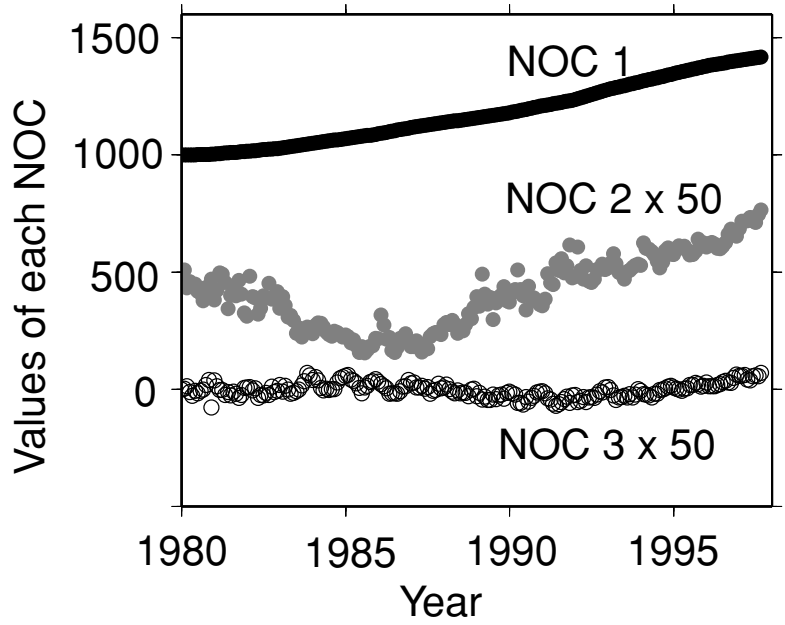

Fig. 4. Three major NOCs - temporal function of the model. NOC2 and NOC3 are magnified by a factor of 50.

\section{Modeling for Japan during 1980-1997 3.1 Data}

We constructed a regional model for the total force field in Japan in order to demonstrate the validity of the procedure described in the previous section and to detect local changes. Data used in the present work were provided by the Geomagnetic Research Group for Earthquake Prediction in Japan. Proton precession magnetometers were deployed at a number of stations in order to detect episodic changes due to tectonic activities under a nation-wide programme of earthquake prediction research since the late 1960s in Japan (Geomagnetic Research Group on Earthquake Prediction, 1973). Added to these were the data from the JMA (the Japan Meteorological Agency) and GSI (the Geographical Survey Institute) observatories. Though there are about 30 sites, some of them are concentrated in too narrow regions. Therefore, only 12 of them were used in this study (Fig. 2).

Data from some observation sites in Japan are contaminated by artificial noises such as the effect of electrical railways. In order to reduce the effect of noise, values taken every 10 minutes during the night (LT 00:40-03:00) were averaged and used as the representative value of the day. Daily values were again averaged to monthly values for the modeling.

\subsection{Result}

The procedure to obtain NOCs was executed for the monthly averaged values. To the interpolate missing values, the procedures described in Section 2.3 were iterated 100 times for each NOC. How the RMS misfit converged to the final value is shown in Fig. 3. Three major NOCs obtained after the iteration procedures are shown in Fig. 4. It should be noted that each NOC does not contain steplike changes that correspond to the period of data missing. The result shown in Fig. 4 convinces us that the procedure worked well, and each NOC is not the false one produced through missing values.

As described in the previous sections, residuals after
$5 \mathrm{nT} / \mathrm{div}$

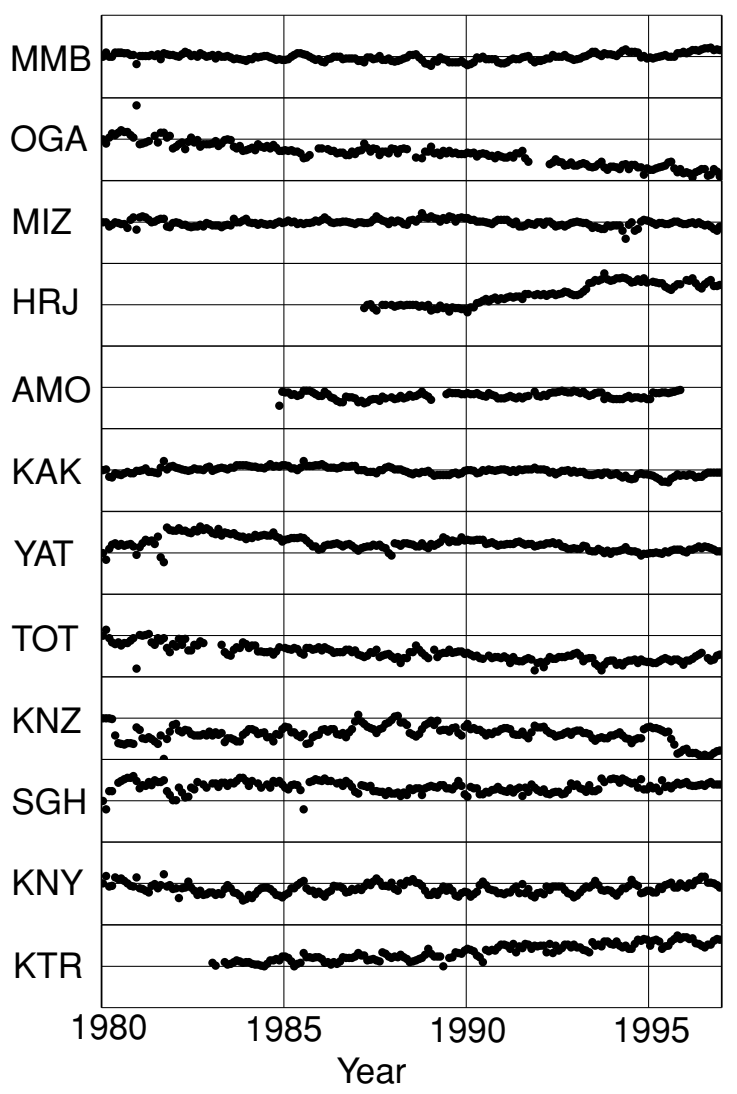

Fig. 5. Residuals after eliminating regional changes represented by three major NOCs.

eliminating the model field are regarded as local changes. Therefore, we will discuss the residual field at each observation site. Residuals at each site are shown in Fig. 5. Some features exist in the residual field. One of the remarkable changes is seen in KNZ after 1995. At this site, the construction of buildings has been ongoing since 1990, and the anomalous changes may be related to this (GSI, personal communications). This anomalous change is also pointed out by the Far East Model (Fujiwara et al., 1998), and some evidence for the effectiveness of both models.

Long-term variations are also seen in some of the stations such as OGA, KTR and TOT. Linear trends are well correlated with NOC1 (Fig. 4), and they should be well explained if no constraints are imposed on the spatial functions. But since we assume the spatial functions can be expressed by Eq. (10), deviated trends are contained in the residual field. Therefore, the linear trends seen in Fig. 5 are regarded as local changes. Some previous studies have indicated that the long-term changes in the geomagnetic field are related to tectonic activities (e.g. Nishida et al., 2004), and the gradual changes seen here may have some meaning in relation to the tectonic information.

Another characteristic, and maybe the most interesting changes, is detected in the data in HRJ. A trend change at 1990 and a step-like change in 1993 can be seen there. Though these features have already been pointed out by other investigators using a simple difference method (e.g. 
Goto et al., 1994; Sakanaka et al., 1996), it is confirmed that these are not false due to unsuitable references, but actual by our generalized approach. In February 1994, an earthquake of M $6.6\left(37^{\circ} 39.2 \mathrm{~N}, 137^{\circ} 18.0 \mathrm{E}\right.$, depth $=$ $24.8 \mathrm{~km}$ ) occurred. Its epicenter is about $20 \mathrm{~km}$ north from the HRJ site, and there may be some relation between the earthquake and the changes in the geomagnetic total force. Changes in geo-electric potential proceeding that earthquake were also reported (Nagao et al., 1996) and an explanation for it was proposed by Ishido (1995). The cause is not clear in present, but these observation facts suggest that some electromagnetic phenomena did exist at the time of the earthquake's occurrence.

\section{Concluding Remarks}

In this paper, we proposed an iterative procedure for calculating NOC in order to avoid the effect of data missing. The method was applied to the monthly averaged values in the geomagnetic total force data in Japan, and some characteristic changes were successfully detected as the residuals in the NOC model. Since missing data often happens in geomagnetic observations, the procedure described here will contribute to geomagnetic field modeling in any region.

Data used in this paper are total force field variations. However, it is well known that amplitudes of changes in the total force differ when sites are in different main field directions. Therefore, models for three components in the geomagnetic field are required for improved study. Recently, 11 sites for observing three components have been installed by GSI in Japan (Tanabe, 1997). The model based on these three components data will be more helpful for the detection of anomalous changes in the geomagnetic field such as those we pointed out in this paper, and will give us a better understanding of tectonomagnetic phenomena.

Acknowledgments. The authors wish to thank the Geographycal Survey Institute, the Japan Meteorological Agency, and Geomagnetic Research Group on Earthquake Prediction for providing geomagnetic data. The authors are also indebted to H. Murakami and the anonymous reviewer for comments which were very fruitful in improving the manuscript. Some of the figures were drawn using Generic Mapping Tools (Wessel and Smith, 1998).

\section{References}

Burdelnaya, I. A., S. V. Filippov, V. P. Golovkov, S. Fujiwara, T. Tanabe, S. Nishi, M. Kaizu, and S. Matsuzaka, Regional orthogonal models of the geomagnetic field changes over the Far East, Earth Planet Space, 51, 287-296, 1999.

Fainberg, E. B., Separation of the geomagnetic field into a nomal and anomalous part, Geomagnetism and Aeronomy, 15, 144-148, 1975.

Fujiwara, S., T. Tanabe, S. Nishi, S. Matsuzaka, V. P. Golovkov, and S. V. Filippov, Modeling of the geomagnetic total force changes in Japan and the Far East, Proc. Conductivity Anomaly Res., pp. 9-16, 1998 (in Japanese).

Fujiwara, S., T. Nishiki, H. Shirai, H. Hamazaki, and V. P. Golovkov, Modeling the daily mean values of regional geomagnetic total force field changes in Japan, Earth Planet Space, 53, 69-73, 2001.

Geomagnetic Research Group on Earthquake Prediction, Observation of geomagnetic total intensity under the project of earthquake prediction research, Report of the Coordinating Committee for Earthquake Prediction, 9, 109-126, 1973.

Golovkov, V. P. and L. R. Newitt, An evaluation of a regional orthogonal field model for Canada with respect to the Canadian geomagnetic reference field, J. Geomag. Geoelectr., 49, 337-346, 1997.

Goto, T., S. Sakanaka, Y. Hori, Y. Wada, N. Sumitomo, and N. Oshiman, Anomalous changes in the geomagnetic total force associated with the occurrence of the Off-Noto Peninsula Earthquake, Annuals. Disas. Prev. Res. Inst., Kyoto Univ., 37 B-1, 281-291, 1994 (in Japanese with English abstract).

Ishido, T., Electro-kinetic phenomena acompanying with water boilingmechanisms on electrokinetic precursor for earthquakes, Proc. Conductivity Anomaly Res., 100-105, 1995 (in Japanese).

Kadokura, S., A new method for data analysis of precise observation of geomagnetic secular variation, Proc. Conductivity Anomaly Res., 151158, 1990, (in Japanese).

Mandea, M. and S. Macmillan, International Geomagnetic Reference Field - the eighth generation, Earth Planet Space, 52, 1119-1124, 2000.

Nagao, T., U. Seiya, Y. Asai, and Y. Kono, Anomalous changes in geoelectric potential preceding four earthquakes in Japan, A Critical Review of VAN, World Scientific, Singapore, pp. 293-300, 1996.

Nishida, Y., Y. Sugisaki, K. Takahashi, M. Utsugi, and H. Oshima, Tectonomagnetic study in the eastern part of Hokkaido, NE Japan: Discrepancy between observed and calculated results, Earth Planet Space, 56, 1049-1058, 2004.

Sakanaka, S., N. Sumitomo, and N. Oshiman, Secular changes in the geomagnetic total force in Hokuriku, Kinki and Chugoku districts, Japan, Annuals. Disas. Prev. Res. Inst., Kyoto Univ., 39 B-1, 273-285, 1996 (in Japanese with English abstract).

Tanabe, T., The continuous observation equipment of geomagnetism installed in the whole country, J. Geograph. Surv. Inst., 87, 4-12, 1997 (in Japanese).

Wessel, P. and W. H. F. Smith, New, improved version of the Generic Mapping Tools released, EOS Trans. AGU, 79, 579, 1998.

K. Yamazaki (e-mail: kenichi@eri.u-tokyo.ac.jp) and N. Oshiman 